\title{
Visual masking and the recovery phenomenon
}

\author{
PETER H. SCHILLER ${ }^{1}$ AND ALAN GREENFIELD \\ MASSACHUSETTS INSTITUTE OF TECHNOLOGY
}

Two experiments were carried out to investigate visual masking and target recovery. In the first experiment it was found that with superimposed disks of various sizes masking increases with increasing target-mask similarity and contour proximity. The results of the second study show that the extent of masking produced by a disk, which is slightly larger than the target, is drastically reduced when the mask in turn is followed by a ring, the inner contour of which is contiguous with the contour of the mask. This "facilitation effect" decreases as the interval between the mask and the third stimulus is increased. Target recovery in this situation appears to be brought about as a result of the reduction or elimination of the contour of the mask, which, as the first study has shown, is critical in bringing about the masking effect. The results are discussed in terms of some of the known physiological properties of the visual system.

Studies investigating backward visual masking have shown that several different kinds of masking phenomena may be distinguished (Raab, 1963; Kahneman, in press; Schiller, 1965, in press). One kind of masking occurs with superimposed stimuli whose contours are not in close proximity with each other. Under such conditions, the masking effect is intensity dependent in that a more intense mask is needed to produce effective interference. The magnitude of masking increases as the interval between the stimuli is decreased. The effect is primarily monocular; interocular presentation yields only a small and sometimes no effect (Boynton, 1961; Schiller, 1965; Schiller, in press).

Masking can also be brought about by patterned stimuli whose contours lie in close proximity with each other. This kind of interference occurs interocularly and can be obtained with stimuli of equal intensities (Schiller, 1965; Sekuler, 1965; Sperling, 1963).

Recently an interesting recovery effect has been reported by a number of investigators that appears to be germane to pattern masking (Dember \& Purcell, 1967; Robinson, 1967, 1968). These investigators have reported that when a third stimulus is introduced following the target and the mask, the target can be recovered. A number of hypotheses have been advanced to explain these findings.

The aim of this study was to investigate one aspect of this recovery phenomenon and to attempt to explain the effect in relation to contour-masking phenomena. Consequently, the first experiment investigated the effect of contour proximity on masking and the second some of the related effects involving target recovery.

\section{EXPERIMENT 1}

It has previously been shown that in pattern masking the magnitude of the interference is a function of contour proximity or stimulus similarity (Schiller, 1965, 1966; Schiller \& Wiener, 1963; Sekuler, 1965; Streicher \& Pollack, 1967). The more similar the configurational characteristics of the target and mask are, the greater the interference appears to be. In order to relate contour masking to recovery phenomena, in this study relatively simple stimuli were employed, and the contours of the masking stimulus were systematically varied relative to the contour of the target.

Subjects

Two paid volunteer Ss with 20/20 vision were used.

\section{Method and Procedure}

The experiment was carried out employing a three-field tachistoscope and a Sky Instrument Timer. The stimuli, which were transilluminated, were mounted on anodized aluminum rotary disks that permitted repeated exact placement of the visual stimuli (Schiller, 1966).

The target was a .25-deg disk of light. Four masking stimuli were used: $\mathbf{M}_{1}=.29, \mathrm{M}_{2}=.32, \mathbf{M}_{3}=.51$, and $\mathbf{M}_{4}=1.02 \mathrm{deg}$ of visual angle. They were disks of light presented concentrically superimposed on the target. The intensity of the target and the masking stimuli was the same throughout, $5 \mathrm{~mL}$. Stimuli were presented at four interstimulus intervals (ISIs), 5, 20, 50, and $100 \mathrm{msec}$. Stimulus duration was $5 \mathrm{msec}$ for the target and $5 \mathrm{msec}$ for the masking stimuli. The fixation field was dark except for two dimly illuminated concentric circles, $2.5 \mathrm{deg}$ and $3.0 \mathrm{deg}$ in diam.

There were a total of 820 trials per $S$. For each of the 16 target and mask presentations (four stimulus sizes and four ISIs) 40 trials were given. In addition, there were 180 catch trials during which the masking stimuli were shown without being preceded by the target.

The Ss were instructed to fixate in the center of the fixation field. Each stimulus presentation was preceded by the $E$ saying "ready." The Ss were told to report the absence or presence of the target by saying "yes" or "no." The Ss were also told that they would see the target approximately one-half the time.

Prior to the experiment each $\mathrm{S}$ was given practice trials for one session. The data were collected over a period of five sessions per S.

\section{Results}

The per cent of correct target detection as a function of ISI and stimulus size is shown in Fig. 1 for each $S$. The results show that masking stimulus size is a critical variable in masking. The large 1.05-deg stimulus produced no interference at all. As the size of the masking stimulus is decreased the extent of masking increases. Increasing the ISI has the general effect of decreasing the interference. There is one reversal for each $S$.

\section{EXPERIMENT 2}

The second experiment was designed to investigate the target recovery effect employing a target and a masking stimulus similar to those used in Experiment 1, and a ring for the third stimulus. It was hypothesized that the target recovery effect is brought about by minimizing the contour of the mask, which, as a result, produces less interference in the perception of the target. According to this hypothesis, contour reduction shoud be optimal at the shortest interval between the mask and the third stimulus and should decrease with increasing interval between these stimuli.

\section{Subjects}

Two paid volunteer Ss with $20 / 20$ vision were used.

\section{Method and Procedure}

The apparatus and viewing conditions were similar to those in Experiment 1 . The target was a .25-deg disk of light. The masking stimulus was a .32-deg disk and the third stimulus was a ring having an inner diameter of $.32 \mathrm{deg}$ and an outer diameter of 

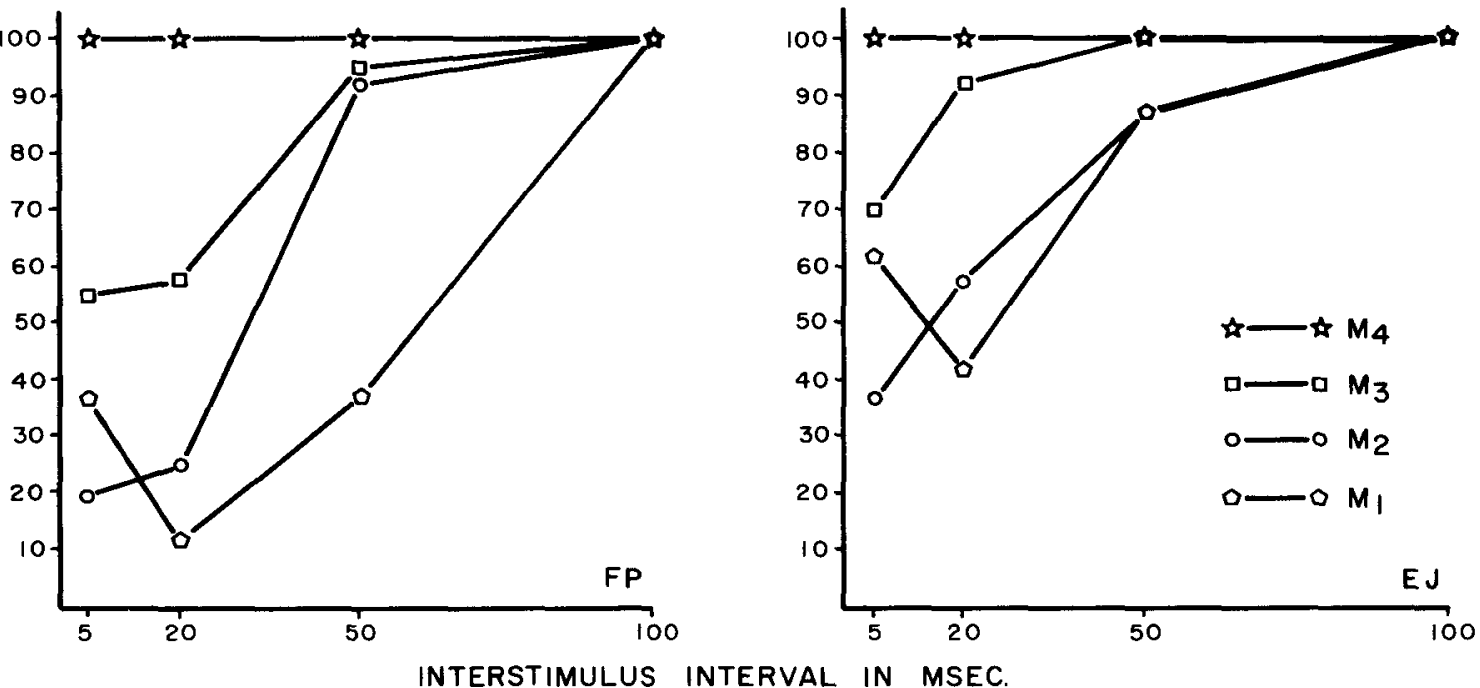

Fig. 1. Per cent correct target detection as a function of masking stimulus size and interstimulus interval. Two Ss, FP and EJ. Target size $=.25 \mathrm{deg}$. Masking stimulus sizes: $M_{4}=1.02 \mathrm{deg} ; M_{3}=.51 \mathrm{deg} ; M_{2}=.32 \mathrm{deg} ; M_{1}=.29 \mathrm{deg}$.

$2.22 \mathrm{deg}$. The stimuli were aligned concentrically. The inner contour of the ring was contiguous with the masking disk. Ss were instructed as in Experiment 1. All stimuli were of equal intensity.

Three intervals were used between the target and the mask $\left(\mathrm{ISI}_{1}\right), 5,20$ and $50 \mathrm{msec}$; the mask and the ring were also presented using three intervals ( $\left.\mathrm{ISI}_{2}\right), 5,50$ and $200 \mathrm{msec}$.

Forty presentations were given for each of these nine conditions per $S$, using all three stimuli. In addition 160 catch trials were given (40 at each ISI) when no target was presented.

\section{Results}

The results of Experiment 2 are shown in Fig. 2. Since the two Ss produced similar results, their data were combined. The results show a very pronounced facilitatory effect at the shortest ISI

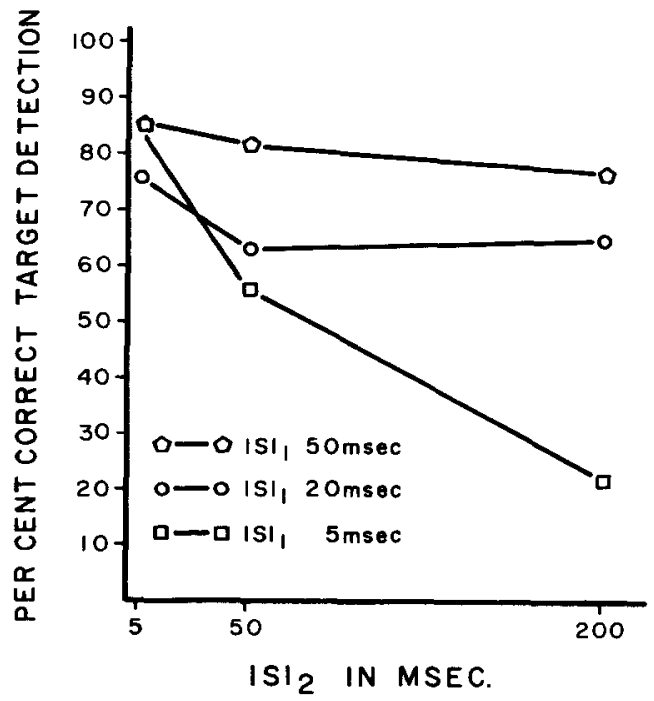

Fig. 2. Per cent correct target detection with three stimuli, target mask and ring. ISI ${ }_{1}$ is interval between target and mask; $\mathrm{ISI}_{2}$ is interval between mask and ring. Target size, $.25 \mathrm{deg}$; mask size, $.32 \mathrm{deg}$. Ring inner diameter, $.32 \mathrm{deg}$; outer diameter, $2.22 \mathrm{deg}$. between the masking stimulus and the third stimulus (ISI $)_{2}$. The extent of this facilitation in the perception of the target decreases as the interval between the mask and the ring is increased. Increasing the interval between the target and the mask also decreases the effect. This latter result is to be expected, since less masking is occurring to begin with.

\section{DISCUSSION}

The results of Experiment 1 have shown that masking with stimuli of equal intensities is contour dependent. Interference is maximal when the contours of the target and mask are in close proximity and decreases as the contours are placed further apart.

The interference effect brought about in backward masking with stimuli of unequal intensities that do not have closely adjacent contours appears to occur as a result of differential processing rate (Schiller, 1968). A dim stimulus elicits discharges in retinal ganglion cells with longer latencies than intense stimuli. The time relationships correspond roughly with the temporal characteristics of masking (Nakayama, 1968; Schiller, 1968, in press).

When stimuli of equal intensities are used, masking occurs only when contours are in close proximity. This effect cannot be explained by an overtake theory, since the processing rate of the two stimuli should be similar. To explain the backward interference effect, it has been hypothesized that information processing rate is limited in the system (Boynton, 1961). In fact, when two stimuli are presented in rapid succession, it has been shown that they fail to produce distinctly separate neural discharges. Furthermore, if the stimuli are similar, the neural responses to the target and mask may not be distinct from the neural responses obtained to the mask alone (Schiller, 1968, in press). It may be assumed that two similar stimuli stimulate identical or closely adjacent receptive fields that fail to generate differential responses to mask alone and mask plus target. Increasing the interval between the stimuli results in the emergence of separate neural responses to each stimulus. Separating the contours of the stimuli brings into play additional receptive fields that may then respond differentially to each of the stimulus contours.

The second experiment has shown that target detection can be improved by the presentation of a third stimulus whose inner contour is contiguous with that of the masking stimulus. The 
effect is optimal at the shortest temporal separation between the mask and third stimulus.

The "facilitation" effect can readily be understood when considered in relation to Experiment 1, where we have seen that the large mask produces little or no masking in contrast to the small one. The effect of introducing a ring following a small mask is to remove the contour of the mask as an effective source of interference and essentially to transform the mask into a large disk.

The response characteristics of single units in the visual system appear to be in concurrence with this view. It has been shown that the majority of cells in the visual system are contour sensitive (Hubel \& Wiesel, 1961, 1965; Kuffler, 1953). Stimulating the center of circular receptive fields of retinal ganglion cells or LGN cells evokes a vigorous response. Stimulating the entire field with diffuse light produces only a small response. In a similar manner, neurons with receptive fields near the border of a stimulus, such as a disk, will fire more than neurons whose receptive fields are within the figure. This effect is brought about by the antagonistic center-surround organization of these units.

The assumed effect of the ring following the mask then is a decrement in the number of discharges of units with their receptive fields at the borders of the mask. Provided the interval between the mask and the ring is short, these units will behave as they do when their entire field is being stimulated; in other words, they respond less to the mask plus ring than to the mask alone. As a result of this, the responses initiated by the target may be more clearly "heard" in the system.

This explanation may also be extended to a situation where the third stimulus is a large disk. One would expect, however, that under such conditions the facilitation should be less. This appears to be the case; the results reported by Robinson $(1967,1968)$ seem to be less drastic than those reported here. Whether or not this kind of explanation is applicable to other kinds of target recovery effects, such as those reported by Dember \& Purcell (1967), remains to be determined.

\section{REFERENCES}

BOYNTON, R. M. Some temporal factors in vision. In W. A. Rosenblith (Ed.), Sensory communication. Cambridge: M.I.T. Press, 1961. Pp. 739-756.
DEMBER, W. N., \& PURCELL, D. G. Recovery of masked visual targets by inhibition of masking stimulus. Science, $1967,157,1335-1336$.

HUBEL, D. H., \& WIESEL, T. N. Integrative action in the cat's lateral geniculate body. Journal of Phy siology, London, 1961, 155, 385-398.

HUBEL, D. H., \& WIESEL, T. N. Receptive fields and functional architecture in two non-striate visual areas (18 and 19) of the cat. Journal of Neurophysiology, 1965, 28, 229-289.

KAHNEMAN, D. Method, findings and theory in studies of visual masking. Psychological Bulletin, 1968, 70, 404-425.

KUFFLER, S. W. Discharge patterns and functional organization of mammalian retina. Journal of Neurophysiology, 1953, 16, 3768.

NAKAYAMA, K. Analysis of backward and forward masking in terms of single unit responses in the cat visual system. Proceedings of the 76th American Psychological Association Convention, 1968, 317-318.

RAAB, D. H. Backward masking. Psychological Bulletin, 1963, 60, 118-129.

ROBINSON, D. N. Visual discrimination of temporal order. Science, 1967, $156,1263-1264$.

ROBINSON, D. N. Visual disinhibition with binocular and interocular presentations. Journal of the Optical Society of America, 1968, 58 , 254-257.

SCHILLER, P. H. Monoptic and dichoptic visual masking by patterns and flashes. Journal of Experimental Psy chology, 1965, 69, 193-199.

SCHILLER, P. H. Forward and backward masking as a function of relative overlap and intensity of test and masking stimuli. Perception \& Psychophysics, 1966, 1, 161-164.

SCHILLER, P. H. Single unit analysis of backward visual masking and metacontrast in the cat lateral geniculate nucleus. Vision Research $1968,8,855-866$.

SCHILLER, P. H. Behavioral and electrophysiological studies of visual masking. Symposium on Information Processing in the Nervous System, Buffalo, in press.

SCHILLER, P. H., \& WIENER, M. Binocular and stereoscopic viewing of geometric illusions. Perceptual \& Motor Skills, 1962, 15, 739-756.

SEKULER, R. W. Spatial and temporal determinants of visual backward masking. Journal of Experimental Psy chology, 1965, 70, $401-406$.

SPERLING, G. What visual masking can tell us about temporal factors in perception. International Congress of Psy chology, 1963, 199-200.

STREICHER, H. W., \& POLLACK, R. H. Backward figure masking as a function of intercontour distance. Psychonomic Science, 1967, 7, 69-70.

\section{NOTE}

1. Address: Department of Psychology, Massachusetts Institute of Technology, Cambridge, Massachusetts 02139 .

(Accepted for publication January 30, 1969.) 